\title{
Expected Attitude Angle Design for Spacecraft Multi-target Tracking*
}

\author{
Yao Zhang ${ }^{1}$, Wei Liu ${ }^{2}$, Jingrui Zhang ${ }^{1}$ \\ ${ }^{1}$ School of Aerospace Engineering, Beijing Institute of Technology, Beijing, China \\ ${ }^{2}$ Technology and Engineering Center for Space Utilization, Chinese Academy of Science, Beijing, China \\ zhangyao7000@yahoo.cn, 1w20060846@yahoo.com.cn, zhangjingrui@bit.edu.cn
}

\begin{abstract}
This paper studies the spacecraft multi-target tracking. Firstly, the spacecraft expected attitude angle is designed according to the mission requirement, namely having the camera on the chaser spacecraft point strictly to the target spacecraft and maximizing the communication time between the antenna on the chaser spacecraft and the Earth. Then, the appropriate antenna installation angle is calculated based on task constraints. Finally, numerical simulation is presented to verify the effectiveness of the design of expected attitude angle and antenna installation angle.
\end{abstract}

Index Terms - Fixed Antenna, Installation Angle Design, Multitarget Tracking, Expected Attitude Angle

\section{Introduction}

Recently, distributed small satellite system becomes research hotspot in the field of international application satellite $^{[1-2]}$, such as TacSat, OE, ORS and F6(Future, Fast, Flexible, Fractionated, Free-Flying). Distributed small satellite system is composed of two or more small satellites on one or more orbits with certain requirements, coordinating and cooperating to complete some space mission and achieve greater value. These small satellites have the feature of small size, light weight, low cost, short development cycle, fast and flexible, timely application of new technologies, etc. However, to achieve high accuracy and fast attitude tracking control of the small satellites, the problem of constraints must be considered during the controller design.

In the field of spacecraft attitude control, the spacecraft multi-target attitude tracking dynamics and control is an important technology. Now the multi-target tracking technology is mostly focus on orbit design ${ }^{[3-4]}$ and navigation algorithm $^{[5]}$. Yet there is few studies on multi-target attitude tracking control ${ }^{[6]}$. In the circumstances that payload on the spacecraft can be maneuvered, multi-target attitude tracking control is achieved by the maneuvers of the spacecraft centerbody and the payload. In the attitude maintaining or tracking control of the spacecraft center-body, the interference caused by the rotational motion of the payload is defined as the external disturbance torque, and robust control or adaptive control method is designed to eliminate the effect of the disturbance torque ${ }^{[7-8]}$.

In this paper, the attitude tracking control problem of some satellite with multi-target tracking constrained in distributed small-satellite system was studied. Due to the limited size, antenna and camera were fixed on the small satellite firmly. The antenna installation angle and the expected attitude angle are designed to ensure that the camera points strictly to the target spacecraft, and the communication time with the Earth could be maximized simultaneously.

\section{Spacecraft Motion Equation}

Some of the coordinate systems in the chaser spacecraft are defined as:

Geocentric inertial coordinate $O X_{i} Y_{i} Z_{i}$, which is the reference coordinate of the system and shortened as $I$; spacecraft body coordinate $O x_{b} y_{b} z_{b}$, shortened as $B$; the attitude angle between coordinates $I$ and $B$ is $\psi, \theta, \varphi$, and the transformation matrix is $A_{B I}$; expected coordinate $O x_{d} y_{d} z_{d}$, shortened as $D$ with its origin point $O$ located at the mass center of the chaser spacecraft. Axis $O x_{d}$ points to the target spacecraft. Axis $O y_{d}$ points to the vector cross direction of $O x_{d}$ and $O x_{b}$. Axis $O z_{d}$ is determined by the Right-hand rule.

Spacecraft attitude kinematics can be described with quaternion as:

$$
\left\{\begin{array}{c}
\dot{q}_{0}=-\frac{1}{2} \vec{q}_{v}^{T} \vec{\omega} \\
\dot{\vec{q}}_{v}=\frac{1}{2}\left(q_{0} I+\vec{q}_{v}^{\times}\right) \vec{\omega}
\end{array}\right.
$$

The error quaternion of coordinate $B$ relative to coordinate $D$ is defined as:

$$
\vec{q}_{e}=\vec{q}_{d}^{*} \otimes \vec{q}=\left[\begin{array}{ll}
q_{e 0} & \vec{q}_{e v}^{T}
\end{array}\right]^{T}=\left[\begin{array}{llll}
q_{e 0} & q_{e 1} & q_{e 2} & q_{e 3}
\end{array}\right]^{T}
$$

The transformation matrix of coordinate $B$ relative to coordinate $D$ can be written as:

$$
A_{B D}=A_{B I}\left(A_{D I}\right)^{T}=\left(q_{e 0}^{2}-\vec{q}_{e v}^{T} \vec{q}_{e v}\right) I+2 \vec{q}_{e v} \vec{q}_{e v}^{T}-2 q_{e 0} \vec{q}_{e v}^{\times}
$$

The error angular velocity of coordinate $B$ relative to coordinate $D$ is defined as:

$$
\vec{\omega}_{e}=\vec{\omega}-A_{B D} \vec{\omega}_{d}
$$

\footnotetext{
* This work is partially supported by National Natural Science Foundation of China \#11172036 and Excellent young scholars Research Fund of Beijing Institute of Technology.
} 
The kinematics of the spacecraft attitude tracking error quaternion can be written as:

$$
\left\{\begin{array}{c}
\dot{q}_{e 0}=-\frac{1}{2} \vec{q}_{e v}^{T} \vec{\omega}_{e} \\
\dot{\bar{q}}_{e v}=\frac{1}{2}\left(q_{e 0} I+\bar{q}_{e v}^{\times}\right) \vec{\omega}_{e}
\end{array}\right.
$$

The spacecraft attitude dynamics can be expressed as:

$$
J \dot{\bar{\omega}}=-\bar{\omega}^{\times} J \vec{\omega}+\vec{T}_{c}+\vec{T}_{d}
$$

\section{Expected Attitude Angle Design}

A. The camera fixed on the chaser spacecraft points to the target spacecraft in real-time

The target spacecraft is designated as satellite $T$, and the chaser spacecraft is designated as satellite $F$. In the process of satellite $F$ real-time pointing to satellite $T$, the target vector $\vec{d}_{F T}$ can be derived from the orbit information of two spacecraft, written as $\vec{d}_{F T I}=\vec{r}_{T I}-\vec{r}_{F I}$, where $\vec{r}_{T I}$ and $\vec{r}_{F I}$ are the radius vector of two spacecraft expressed under coordinate I. $\vec{u}_{F T}$ is the unit vector of $\vec{d}_{F T}$, called the unit direction vector and written as $\vec{u}_{F T}=\vec{d}_{F T} /\left|\vec{d}_{F T}\right|$.

When satellite $F$ is in the expected attitude, the sight axis of the camera points exactly to satellite $T$, and the projection array of $\vec{u}_{F T}$ in the coordinate $D$ of satellite $F$ is $\vec{u}_{F T D}=\left[\begin{array}{lll}1 & 0 & 0\end{array}\right]^{T}$. With the $\vec{u}_{F T}$ expressed under coordinate $I$ written as $\vec{u}_{F T I}, \vec{u}_{F T I}=A_{D I}^{T} \vec{u}_{F T D}$ is obtained. The expected pitch angle and yaw angle can be written respectively as:

$$
\begin{aligned}
& \theta_{d}=-\arcsin \left(u_{F T I z}\right) \\
& \psi_{d}=\arctan \left(\frac{u_{F T I y}}{u_{F T I x}}\right)
\end{aligned}
$$

When the camera points to satellite $T$ in real-time, the roll angle around the body axis $O x_{b}$ is unconstrained. The roll angle can then be controlled to achieve maximum communication time with the Earth.

\section{B. Chaser spacecraft communicates with the Earth}

As mentioned above, the antenna is fixed on satellite $F$ and can't rotate. To make sure that satellite $F$ communicates with the Earth, the shaft of the antenna should point to the Earth.

Firstly, design the optimal antenna installation angle $(\beta, \gamma)$. By projecting the unit vector of antenna shaft $\vec{u}_{a}$ into $O x_{b} y_{b}$, the angle between the projection and the positive direction of $O x_{b}$ is defined as $\beta(0 \leq \beta \leq 2 \pi) . \gamma(0 \leq \gamma \leq \pi)$ is the angle between $\vec{u}_{a}$ and the positive direction of $O z_{b}$. To reduce the maneuver process, roll angular velocity and initial roll attitude angle can be set at zero when designing the antenna installation angle, written as $\varphi_{d}=0, \dot{\varphi}_{d}=0$.
Define $-\vec{u}_{F I}$ as the unit vector of satellite $F$ pointing in the direction of the Earth. When satellite $F$ communicates with the Earth, the maximum $\sigma$ can be written as:

$$
\sigma_{\max }=\arcsin \frac{R}{\left|\vec{r}_{F I}\right|}=\arcsin \frac{R}{\sqrt{r_{F I x}^{2}+r_{F I y}^{2}+r_{F I z}^{2}}}
$$

$\vec{u}_{a}$ is expressed under coordinate $D$ as $\vec{u}_{a D}=\left[\begin{array}{lll}\sin \gamma \cos \beta & \sin \gamma \sin \beta & \cos \gamma\end{array}\right]^{T}$, and expressed under coordinate $I$ as:

$$
\begin{aligned}
& \vec{u}_{a I}=\left(A_{D I}\right)^{T} \vec{u}_{a D} \\
& =\left[\begin{array}{c}
\cos \theta_{d} \cos \psi_{d} \sin \gamma \cos \beta \\
\cos \theta_{d} \sin \psi_{d} \sin \gamma \cos \beta \\
-\sin \theta_{d} \sin \gamma \cos \beta
\end{array}\right] \\
& +\left[\begin{array}{c}
\sin \theta_{d} \cos \psi_{d} \sin \gamma \sin \beta+\sin \psi_{d} \cos \gamma \\
\sin \theta_{d} \sin \psi_{d} \sin \gamma \sin \beta-\cos \psi_{d} \cos \gamma \\
\cos \theta_{d} \sin \gamma \sin \beta
\end{array}\right] \sin \varphi_{d} \\
& +\left[\begin{array}{c}
-\sin \psi_{d} \sin \gamma \sin \beta+\sin \theta_{d} \cos \psi_{d} \cos \gamma \\
\cos \psi_{d} \sin \gamma \sin \beta+\sin \theta_{d} \sin \psi_{d} \cos \gamma \\
\cos \theta_{d} \cos \gamma
\end{array}\right] \cos \varphi_{d}
\end{aligned}
$$

When $\varphi_{d}==0, \vec{u}_{a I}$ could be expressed as:

$$
\begin{aligned}
\vec{u}_{a I 0} & =\left[\begin{array}{c}
\cos \theta_{d} \cos \psi_{d} \sin \gamma \cos \beta-\sin \psi_{d} \sin \gamma \sin \beta \\
\cos \theta_{d} \sin \psi_{d} \sin \gamma \cos \beta+\cos \psi_{d} \sin \gamma \sin \beta \\
-\sin \theta_{d} \sin \gamma \cos \beta
\end{array}\right] \\
+ & {\left[\begin{array}{c}
+\sin \theta_{d} \cos \psi_{d} \cos \gamma \\
\sin \theta_{d} \sin \psi_{d} \cos \gamma \\
\cos \theta_{d} \cos \gamma
\end{array}\right] }
\end{aligned}
$$

Define $\sigma_{0}$ as the angle between $\vec{u}_{a}$ and $-\vec{u}_{F I} \cdot \sigma_{0}$ represents the communication ability and is expressed as:

$$
\begin{aligned}
\cos \sigma_{0}=\frac{\vec{u}_{a I 0} \cdot\left(-\vec{u}_{F I}\right)}{\left|\vec{u}_{a I 0}\right|\left|-\vec{u}_{F I}\right|} \\
=-u_{F I x}\left(\cos \theta_{d} \cos \psi_{d} \sin \gamma \cos \beta-\sin \psi_{d} \sin \gamma \sin \beta\right. \\
\left.\quad+\sin \theta_{d} \cos \psi_{d} \cos \gamma\right) \\
\quad-u_{F I y}\left(\cos \theta_{d} \sin \psi_{d} \sin \gamma \cos \beta+\cos \psi_{d} \sin \gamma \sin \beta\right. \\
\left.\quad+\sin \theta_{d} \sin \psi_{d} \cos \gamma\right) \\
\quad-u_{F I z}\left(-\sin \theta_{d} \sin \gamma \cos \beta+\cos \theta_{d} \cos \gamma\right)
\end{aligned}
$$

Based on $0 \leq \sigma_{0} \leq \sigma_{\max }$, the optimal installation angle $(\beta, \gamma)$ can be obtained by numerical calculation. Firstly, discrete $\beta(0 \leq \beta \leq 2 \pi)$ and $\gamma(0 \leq \gamma \leq \pi)$. Here $\beta(0 \leq \beta \leq 2 \pi)$ is divided into 101 points by interval $2 \pi / 100$, and $\gamma(0 \leq \gamma \leq \pi)$ is divided into 101 points by interval $\pi / 100$. So the error of the installation angle is less than $3^{\circ}$. Then take 
over all the discrete points and calculate to see if the communication constraint could be satisfied during two orbital periods. Then the optimal antenna installation angle $(\beta, \gamma)$ is obtained.

As is required, the antenna of the chaser spacecraft should point to the Earth, and the roll angle should ensure the maximum communication time with the Earth. The angle $\sigma$ between $\vec{u}_{a}$ and $-\vec{u}_{F I}$ is written as:

$$
f\left(\varphi_{d}\right)=\cos \sigma=\frac{\vec{u}_{a I} \cdot\left(-\vec{u}_{F I}\right)}{\left|\vec{u}_{a I}\right|\left|-\vec{u}_{F I}\right|}=a \sin \varphi_{d}+b \cos \varphi_{d}+c(
$$

where,

$a=-u_{F I x}\left(\sin \theta_{d} \cos \psi_{d} \sin \gamma \sin \beta+\sin \psi_{d} \cos \gamma\right)-u_{F I y}\left(\sin \theta_{d}\right.$ $\left.\sin \psi_{d} \sin \gamma \sin \beta-\cos \psi_{d} \cos \gamma\right)-u_{F I z}\left(\cos \theta_{d} \sin \gamma \sin \beta\right)$ $b=-u_{F I x}\left(-\sin \psi_{d} \sin \gamma \sin \beta+\sin \theta_{d} \cos \psi_{d} \cos \gamma\right)-u_{F I y}($ $\left.\cos \psi_{d} \sin \gamma \sin \beta+\sin \theta_{d} \sin \psi_{d} \cos \gamma\right)-u_{F I z}\left(\cos \theta_{d} \cos \gamma\right)$ $c=-u_{F I x}\left(\cos \theta_{d} \cos \psi_{d} \sin \gamma \cos \beta\right)-u_{F I y}\left(\cos \theta_{d} \sin \psi_{d}\right.$ $\sin \gamma \cos \beta)-u_{F I z}\left(-\sin \theta_{d} \sin \gamma \cos \beta\right)$

To achieve the communication with the Earth, roll angle $\varphi_{d}$ should be designed to minimize $\sigma$, and the roll angle $\varphi_{d}$ should be designed to maximize $f\left(\varphi_{d}\right)$. Define $\delta$ as:

$$
\sin \delta=\frac{|b|}{\sqrt{a^{2}+b^{2}}}
$$

While $a \geq 0, b>0, f\left(\varphi_{d}\right)=\cos \sigma=\sqrt{a^{2}+b^{2}} \sin \left(\varphi_{d}+\delta\right)+c$, $\varphi_{d}$ can be written as $\varphi_{d}^{*}=\pi / 2-\delta$.

While $a \geq 0, b \leq 0, f\left(\varphi_{d}\right)=\cos \sigma=\sqrt{a^{2}+b^{2}} \sin \left(\varphi_{d}-\delta\right)+c$, $\varphi_{d}$ can be written as $\varphi_{d}^{*}=\pi / 2+\delta$.

While $a<0, b \geq 0, f\left(\varphi_{d}\right)=\cos \sigma=\sqrt{a^{2}+b^{2}} \sin \left(-\varphi_{d}+\delta\right)+c$, $\varphi_{d}$ can be written as $\varphi_{d}^{*}=-\pi / 2+\delta$.

While $a<0, b<0, f\left(\varphi_{d}\right)=\cos \sigma=-\sqrt{a^{2}+b^{2}} \sin \left(\varphi_{d}+\delta\right)+c$, $\varphi_{d}$ can be written as $\varphi_{d}^{*}=-\pi / 2-\delta$.

Then the expected attitude angle of satellite $F$ could be described as:

$$
\begin{aligned}
& \varphi_{d}^{*}= \begin{cases}\pi / 2-\delta & a \geq 0, b>0 \\
\pi / 2+\delta & a \geq 0, b \leq 0 \\
-\pi / 2+\delta & a<0, b \geq 0 \\
-\pi / 2-\delta & a<0, b<0\end{cases} \\
& \theta_{d}=-\arcsin u_{B A Z z} \quad \psi_{d}=\arctan \left(u_{B A y y} / u_{B A I x}\right)
\end{aligned}
$$

According to the expected Euler angle, $\vec{q}_{d}$ could be expressed as:

$$
\begin{aligned}
\vec{q}_{d}=\left[\begin{array}{l}
q_{d 0} \\
q_{d 1} \\
q_{d 2} \\
q_{d 3}
\end{array}\right] & =\left[\begin{array}{l}
\cos \left(\psi_{d} / 2\right) \cos \left(\theta_{d} / 2\right) \cos \left(\varphi_{d}^{*} / 2\right) \\
\cos \left(\psi_{d} / 2\right) \cos \left(\theta_{d} / 2\right) \sin \left(\varphi_{d}^{*} / 2\right) \\
\cos \left(\psi_{d} / 2\right) \sin \left(\theta_{d} / 2\right) \cos \left(\varphi_{d}^{*} / 2\right) \\
\sin \left(\psi_{d} / 2\right) \cos \left(\theta_{d} / 2\right) \cos \left(\varphi_{d}^{*} / 2\right)
\end{array}\right] \\
& +\left[\begin{array}{c}
\sin \left(\psi_{d} / 2\right) \sin \left(\theta_{d} / 2\right) \sin \left(\varphi_{d}^{*} / 2\right) \\
-\sin \left(\psi_{d} / 2\right) \sin \left(\theta_{d} / 2\right) \cos \left(\varphi_{d}^{*} / 2\right) \\
\sin \left(\psi_{d} / 2\right) \cos \left(\theta_{d} / 2\right) \sin \left(\varphi_{d}^{*} / 2\right) \\
-\cos \left(\psi_{d} / 2\right) \sin \left(\theta_{d} / 2\right) \sin \left(\varphi_{d}^{*} / 2\right)
\end{array}\right]
\end{aligned}
$$

\section{Numerical Simulation}

Six orbital elements of satellite $T$ and satellite $F$, and the initial attitude angle and initial angular velocity of satellite $F$ can be given as follows:

$$
\left\{\begin{array}{l}
a_{T}=8000 \mathrm{~km} \\
e_{T}=0.15 \\
\Omega_{T}=45^{\circ} \\
i_{T}=30^{\circ} \\
\omega_{T}=30^{\circ} \\
t_{p T}=0 s
\end{array},\left\{\begin{array}{l}
a_{F}=8000 \mathrm{~km} \\
e_{F}=0.13 \\
\Omega_{F}=44^{\circ} \\
i_{F}=31^{\circ} \\
\omega_{F}=29^{\circ} \\
t_{p F}=2 s
\end{array},\left\{\begin{array}{l}
\varphi_{0}=30^{\circ} \\
\theta_{0}=30^{\circ} \\
\psi_{0}=30^{\circ} \\
\omega_{x}=0.001 \mathrm{rad} / \mathrm{s} \\
\omega_{y}=0.001 \mathrm{rad} / \mathrm{s} \\
\omega_{z}=0.001 \mathrm{rad} / \mathrm{s}
\end{array}\right.\right.\right.
$$

The control law is as follows ${ }^{[9]}$

$$
\vec{T}_{c}=J_{0}\left(-\bar{\omega}_{e}^{\times} A_{B D} \bar{\omega}_{d}+A_{B D} \dot{\bar{\omega}}_{d}\right)+\bar{\omega}^{\times} J_{0} \bar{\omega}-D \vec{\omega}_{e}-K \vec{q}_{e}
$$

Other parameter values can be selected as follows: $\mu_{e}=3.986 \times 10^{5} \mathrm{~km}^{3} / \mathrm{s}^{2}, R=6378 \mathrm{~km}, K=\operatorname{diag}(1.8,1.8,1.4)$, $D=\operatorname{diag}(4,4,2), J=\operatorname{diag}[20,20,10] \mathrm{kg} \cdot \mathrm{m}^{2}$. The simulation time is two orbital periods (14242 seconds). The results are as follows.

Fig. 1 shows the communication time distribution according to the different antenna installation angle during the two orbital periods. Optimal installation angle observed in the simulation is $\beta=5.1522 \mathrm{rad}, \gamma=1.6022 \mathrm{rad}$, and the communication time is $10048 \mathrm{~s}$. Fig. 2 is the antenna installation diagram.

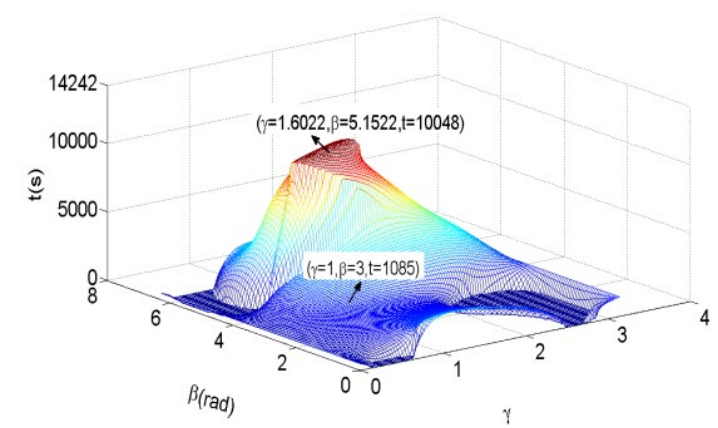

Fig. 1. Communication time distribution of different installation angle 


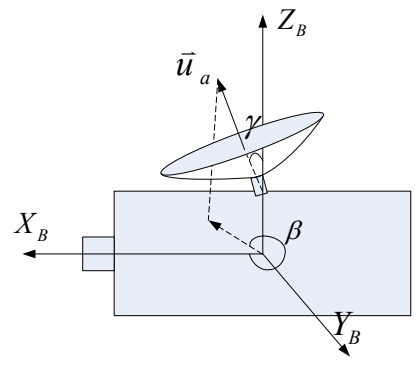

Fig. 2. Antenna installation diagram

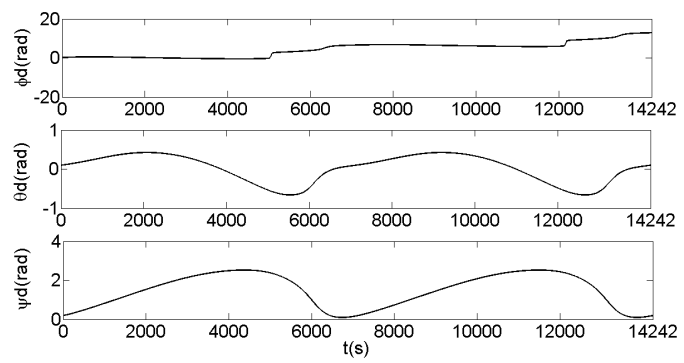

Fig. 3. Expected attitude angle under multi-target tracking condition

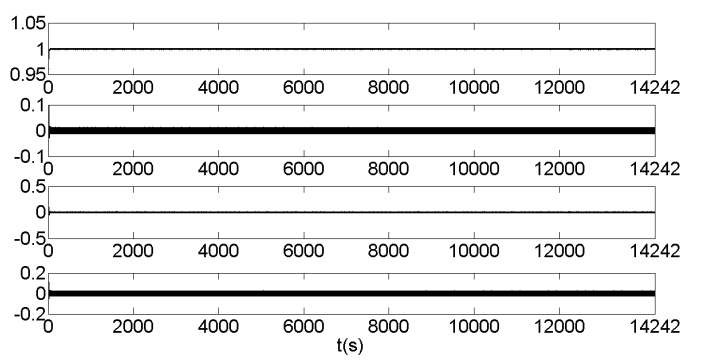

Fig. 4. Error quaternion of pointing and tracking

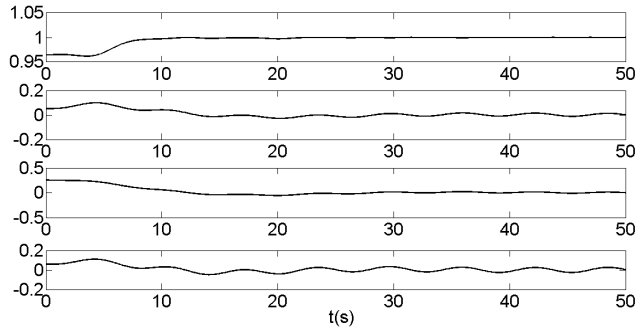

Fig. 5. The history of error quaternion for pointing and tracking in the first 50 seconds

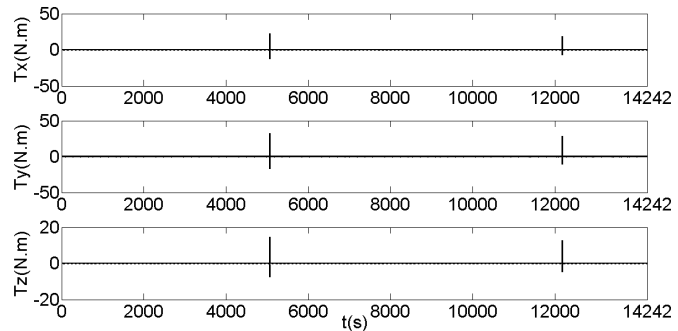

Fig. 6. Control torques of spacecraft under multi-target tracking condition

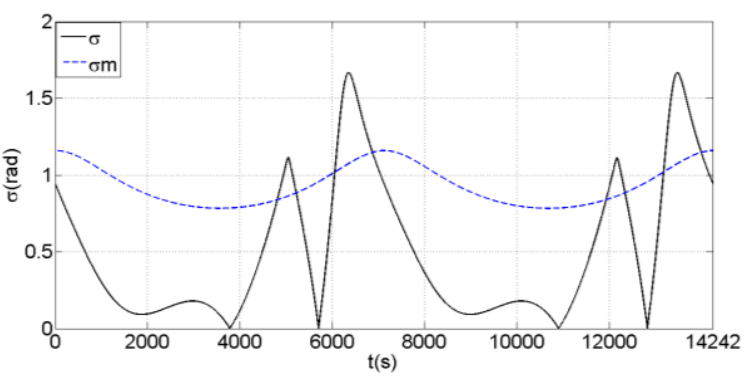

Fig. 7. Communication ability with $\beta=5.1522 \mathrm{rad}, \gamma=1.6022 \mathrm{rad}$

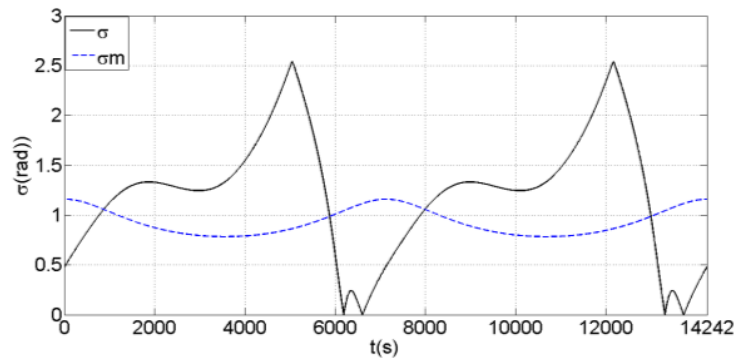

Fig. 8. Communication ability with $\beta=3 \mathrm{rad}, \gamma=1 \mathrm{rad}$

Fig. 3 outlines the history of the spacecraft expected attitude angle under the multi-target tracking condition. As required, the camera on satellite $F$ should track satellite $T$ in real-time, and simultaneously keep the antenna pointing to the earth for communication. Fig. 5 shows that the tracking is achieved at about the 20th second, and is maintained during the whole period (see Fig. 4).

Fig. 7 and Fig. 8 show the ability of satellite $F$ to communicate with the Earth. The dashed line in the figures represents the history of $\sigma_{\max }$. The solid line represents the real $\sigma$ with different installation angle. $\sigma$ under $\sigma_{\max }$ suggests that the chaser spacecraft could achieve communication with the Earth. In Fig. 7, the communication time is $11805 \mathrm{~s}$ with the installation angle $\beta=5.1522 \mathrm{rad}$, $\gamma=1.6022 \mathrm{rad}$, while in Fig. 8, the communication time is $4237 \mathrm{~s}$ with the installation angle $\beta=3 \mathrm{rad}, \gamma=1 \mathrm{rad}$.

The significant difference between Fig. 7 and Fig. 8 verifies the necessity of the installation angle design for the antenna.

\section{Conclusions}

This paper studied the multi-target tracking control for spacecraft with fixed antenna. The chaser spacecraft is expected to track the target satellite and orient to the Earth at the same time. Two constraint conditions, therefore, have to be met by the chaser spacecraft. Firstly, the camera must track the target in real-time; secondly, the shaft of the antenna should point to the Earth for communication. In the study, both the installation angle for the fixed-antenna and the optimal expected attitude angle were taken into consideration simultaneously. 
Compared with the movable antenna, the antenna fixed into spacecraft comprises less moving components, thus reducing the disturbance caused by these components. The simulation results verified the effectiveness of the design.

The method used in designing the expected attitude angle could be applied to the design of the attitude tracking controller for other multi-target tasks and has great potential applicability in engineering.

\section{References}

[1] Maurice, M. and Jeff, S. and James, L.: AFRL plug-and-play Spacecraft Avionics Experiment (SAE), Aerospace Conference, 2012 IEEE, pp.1-6.

[2] Bridges, C.P. and Vladimirova, T.: Real-time agent middleware experiments on java-based processors towards distributed satellite systems, Aerospace Conference, 2011 IEEE, pp.1 - 10.

[3] Barbee, B.W. and Alfano, S. and Pinon, E. and Gold, K. and Gaylor, D.: Design of Spacecraft Missions to Remove Multiple Orbital Debris Objects, Aerospace Conference, IEEE, 2011, pp.1-14.
[4] Jared Wood and J. Karl Hedrick: Space Partitioning and Classification for Multi-target Search and Tracking by Heterogeneous Unmanned Aerial System Teams, AIAA, 2011, pp.1-11.

[5] Nie Hongshan and Huang Zhijian and Diao Jietao and Li Qiang and Yu Hongqi and Yi Wei: Research on Space-Based Space Target Detecting and Tracking Algorithm, International Conference on Intelligent System Design and Engineering Application, 2010, pp.253-256.

[6] Masaki Takahashi and Yasuyuki Nanamori and Kazuo Yoshida: Rapid Multi-target Pointing and High Accuracy Attitude Control Steering Law of Variable Speed Control Moment Gyroscopes, AIAA Guidance, Navigation and Control Conference and Exhibit, 2008, pp.1-10.

[7] Yeh, F.-K., "Sliding-mode adaptive attitude controller design for spacecrafts with thrusters", Control Theory \& Applications, IET, 4(2010), pp.1254-1264.

[8] An-Min Zou and Kumar, K.D. and Zeng-Guang Hou: Quaternion-Based Adaptive Output Feedback Attitude Control of Spacecraft Using Chebyshev Neural Networks, Neural Networks, IEEE Transactions on, 21(2010), pp.1457-1471.

[9] Matthew C, VanDyke: Decentralized Coordinated Attitude Control of a Formation of Spacecraft, The Virginia Polytechnic Institute and State University, 